\title{
EVERY ISOMETRY IS REFLEXIVE
}

\author{
JAMES A. DEDDENS ${ }^{1}$
}

Abstract. A bounded linear operator $A$ on a Hilbert space $\mathfrak{H C}$ is called reflexive if any bounded linear operator which leaves invariant the invariant subspaces of $A$ is a limit of polynomials in $A$ in the weak operator topology. In this note we prove that every isometry $V$ on a Hilbert space $\mathcal{H}$ is reflexive.

Let $A$ be a bounded linear operator on a Hilbert space $\Re$. We denote by $\operatorname{Lat}(A)$ the set of all closed subspaces $\mathfrak{T}$ of $\mathcal{H}$ such that $A \mathfrak{N} \subseteq \mathfrak{T}$, and by $\mathrm{Q}_{A}$ the smallest weakly closed algebra containing $A$ and $I$ (i.e. the closure in the weak operator topology of $p(A)$ for all polynomials $p) . A$ is called reflexive if, for $B$ a bounded linear operator on $\mathfrak{H C}$, Lat $(A) \subseteq$ Lat $(B)$ implies $B \in \mathfrak{Q}_{\boldsymbol{A}}$.

D. Sarason in [4] proved that every normal (and hence every unitary) operator is reflexive, and that the unilateral shift of multiplicity one is also reflexive. Our task is to piece together these two results to show that every isometry is reflexive. We might mention that it is unknown whether the direct sum of two arbitrary reflexive operators is reflexive.

Let $V$ be an isometry on $\mathfrak{F C}$ (i.e. $\|V x\|=\|x\|$ for all $x \in \mathcal{F}$ ). Then there exist unique reducing subspaces $\mathfrak{T}_{\infty} \equiv \bigcap_{n=0}^{\infty} V^{n} \mathfrak{H}$ and $\mathfrak{T}_{+}$ $\equiv \sum_{n=0}^{\infty} \oplus V^{n}(\mathfrak{H C} \ominus V \mathfrak{H C})$ such that $\mathfrak{H}=\mathfrak{M}_{\infty} \oplus \mathfrak{M}_{+}$with $U=\left.V\right|_{\mathfrak{M}_{\infty}}$ a unitary operator and $U_{+}=\left.V\right|_{\Re_{+}}$a unilateral shift [3, Problem 118]. If we let $E(\cdot)$ be the spectral measure of $U$ and $\mathfrak{T}_{s} \equiv\left\{x \in \mathfrak{N}_{\infty}\right.$ : $\|E(\cdot) x\| \perp$ Leb $\}$ and $\mathfrak{M}_{a} \equiv\left\{x \in \mathfrak{M}_{\infty}:\|E(\cdot) x\| \ll\right.$ Leb $\}$ then $\mathfrak{M}_{\infty} \equiv \mathfrak{M}_{s}$ $\oplus \mathscr{N}_{a}$ with $U_{s}=\left.U\right|_{\mathscr{N}_{c}}$ and $U_{a}=\left.U\right|_{\mathscr{N}_{a}}$ being called the singular and absolutely continuous parts of $U$ respectively [2].

ThEOREM. Every isometry $V=U_{\mathbf{s}} \oplus U_{a} \oplus U_{+}$on a Hilbert space $\nVdash$ is reflexive.

Before we prove this result we need some lemmas, which are of interest in their own right.

Lemma 1. $\operatorname{Lat}\left(U_{s} \oplus U_{a}\right)=\operatorname{Lat}\left(U_{s}\right) \oplus \operatorname{Lat}\left(U_{a}\right)$.

Received by the editors June $18,1970$.

AMS 1969 subject classifications. Primary 4710, 4735, 4740.

Key words and phrases. Weakly closed unstarred operator algebras, invariant and reducing subspaces, absolutely continuous and singular unitary operators, unilateral and bilateral shifts.

1 This research was partially supported by the National Science Foundation. 
Proof. We need only show that $\mathfrak{T C} \in \operatorname{Lat}\left(U_{s} \oplus U_{a}\right)$ implies $\mathfrak{T}$ $=P_{\mathbb{M}_{\mathfrak{s}}} \mathfrak{T} \oplus P_{\mathbb{N}_{a}} \mathfrak{M}$. First suppose $\mathfrak{T}$ reduces $U_{\mathfrak{s}} \oplus U_{a}$. Then $P_{\mathfrak{N}}$ commutes with $U_{s} \oplus U_{a}$ or matricially,

$$
P_{\mathfrak{N}}=\left(\begin{array}{ll}
A & B \\
B^{*} & C
\end{array}\right) \text { commutes with }\left(\begin{array}{ll}
U_{*} & 0 \\
0 & U_{a}
\end{array}\right) .
$$

So $B U_{a}=U_{a} B$. By Theorem 3 in [1] we note that $B=0$. Hence $P_{\mathfrak{M}}=A \oplus C$ or $\mathfrak{T}=P_{\Re_{s}} \mathfrak{T} \oplus P_{\mathfrak{M}_{a}} \mathfrak{T}$. Now suppose $\mathfrak{T} \in \operatorname{Lat}\left(U_{s} \oplus U_{a}\right)$ with $U=U_{s} \oplus U_{a}$. If $\mathscr{L}=\mathfrak{M} \ominus U \mathfrak{T}$ then

$$
\mathfrak{N}=\sum_{n=0}^{\infty} \oplus U^{n} \mathscr{L} \oplus \bigcap_{n=0}^{\infty} U^{n} \mathfrak{T l} \equiv \Re \oplus R,
$$

where $R$ reduces $U$. Hence $R=P_{\mathfrak{M}_{a}} R \oplus P_{\mathbb{N}_{a}} R$. Since

$$
\mathfrak{N}=\sum_{n=0}^{\infty} \bigoplus U^{n} \mathfrak{L} \subseteq \sum_{n=-\infty}^{\infty} \bigoplus U^{n} \mathfrak{L},
$$

and since the bilateral shift is an absolutely continuous unitary, we must have that $\Re \subseteq \Re_{a}$, so $\Re=P_{\Re_{s}} \Re \oplus P_{\Re_{a}} \Re$. Thus $\mathscr{N}=\Re \oplus R$ $=P_{\Re_{s}} \Re \oplus P_{\Re_{a}} \Re$.

Lemma 2. $a_{U_{a} \oplus U_{a}}=a_{U} \oplus a_{U_{a}}$.

Proof. We need only show that $B_{1} \in Q_{U}$ and $B_{2} \in Q_{U_{a}}$ imply $B_{1} \oplus B_{2} \in Q_{U_{s} \oplus U_{a}}$. Since $B_{1} \in Q_{U_{s}}$ and $B_{2} \in Q_{U_{a}}, \operatorname{Lat}\left(U_{s}\right) \subseteq \operatorname{Lat}\left(B_{1}\right)$ and $\operatorname{Lat}\left(U_{a}\right) \subseteq \operatorname{Lat}\left(B_{2}\right)$. Hence $\operatorname{Lat}\left(U_{s}\right) \oplus \operatorname{Lat}\left(U_{a}\right) \subseteq \operatorname{Lat}\left(B_{1} \oplus B_{2}\right)$. Now Lemma 1 implies that $\operatorname{Lat}\left(U_{s} \oplus U_{a}\right) \subseteq \operatorname{Lat}\left(B_{1} \oplus B_{2}\right)$. Since $U_{s} \oplus U_{a}$ is unitary, it is reflexive by Sarason's result [4], and hence $B_{1} \oplus B_{2}$ $\in a_{U_{\bullet} \oplus U_{a}}$.

Corollary. $Q_{U_{s} \oplus U_{a} \oplus U_{+}}=a_{U_{s}} \oplus a_{U_{a} \oplus U_{+}}$.

Proof. We need only show that $B_{1} \in Q_{U_{s}}$ and $B_{2} \in Q_{U_{a} \oplus U_{+}}$imply $B_{1} \oplus B_{2} \in Q_{U_{s} \oplus\left(U_{a} \oplus U_{+}\right)}$. Let $W$ be the bilateral shift on $\Re_{+}$which uniquely extends the unilateral shift $U_{+}$, i.e. $\Re_{+}=\bigvee_{n=-\infty}^{\infty} W^{n} \mathfrak{M}_{+}$and $U_{+}=W \mid \Re_{+}$. Then there is a unique $\tilde{B}_{2} \in Q_{U_{a} \oplus W}$ such that $B_{2}$ $=\widetilde{B}_{2} \mid \mathscr{N}_{a} \oplus \mathscr{N}_{+}$, in fact if $B_{2}=\psi\left(U_{a} \oplus U_{+}\right)$for $\psi \in H^{\infty}$ then $\widetilde{B}_{2}=\psi\left(U_{a} \oplus W\right)$. Since $U_{a} \oplus W$ is an absolutely continuous unitary, Lemma 2 implies that $B_{1} \oplus \widetilde{B}_{2} \in Q_{U_{a} \oplus\left(U_{a} \oplus W\right)}$. Hence $B_{1} \oplus B_{2} \in Q_{U_{\bullet} \oplus\left(U_{a} \oplus U_{+}\right)}$.

Lemma 3. $U_{a} \oplus U_{+}$is reflexive.

Proof. The proof can be simplified if we make the following observation: If $A$ is reflexive and $\left\{\Re_{\alpha}\right\}$ is any collection of invariant subspaces for $A$, then $\left.A \oplus \sum \oplus A\right|_{\Re_{\alpha}}$ is reflexive if for every $T \in Q_{A}$ 
there is a sequence of polynomials $\left\{p_{n}\right\}_{1}^{\infty}$ such that $p_{n}(A) \rightarrow T$ in the weak operator topology and $\left\|p_{n}(A)\right\| \leqq K$ for all $n$. Using the multiplicity theory for absolutely continuous unitaries [2] and unilateral shifts [3], this observation reduces the proof to showing that $M_{e} \oplus M_{e}$ on $L^{2}(E) \oplus H^{2},(E$ a closed subset of $\{z:|z|=1\})$, is reflexive and satisfies the boundedness condition (here $\left(M_{e} f\right)\left(e^{i \theta}\right)=e^{i \theta} f\left(e^{i \theta}\right)$ for $f \in L^{2}(E)$ or $\left.H^{2}\right)$.

Suppose $\operatorname{Lat}\left(M_{e} \oplus M_{e}\right) \subseteq \operatorname{Lat}(B)$. Then $B=B_{1} \oplus B_{2}$ with $\operatorname{Lat}\left(M_{e}\right)$ $\subseteq$ Lat $\left(B_{2}\right)$. Since the unilateral shift $M_{e}$ on $H^{2}$ is reflexive [4], $B_{2} \in Q_{M_{e}}$. So $B_{2}=M_{\psi}, \psi \in H^{\infty}$. By considering the $M_{e} \oplus M_{e}-$ invariant subspaces of the form

$$
\mathscr{M}_{n}=\left\{\left(\left.M_{e}^{*^{n}} \phi\right|_{E}, \phi\right): \phi \in H^{2}\right\}
$$

we conclude that $\left.B_{1} M_{e}^{* n} \phi\right|_{E}=\left.M_{e}^{* n} \psi \phi\right|_{E}$ for all $\phi \in H^{2}$ and $n \geqq 0$. Since $\left\{\left.M_{\theta}^{* n} \phi\right|_{E}: \phi \in H^{2}, n \geqq 0\right\}$ is dense in $L^{2}(E), B_{1}=M_{\left.\psi\right|_{E}}$. Thus $B_{1} \oplus B_{2}$ $=M_{\psi \mid s} \oplus M_{\psi}$ for $\psi \in H^{\infty}$. Hence $B_{1} \oplus B_{2} \in Q_{M_{e} \oplus M_{e}}$, and $M_{e} \oplus M_{e}$ is reflexive. Suppose $T \in Q_{M_{\bullet} \oplus M_{e}}$. Then $T=T_{1} \oplus T_{2}$ where $T_{2} \in Q_{M_{c}}$ on $H^{2}$, so $T_{2}=M_{\psi}$ for $\psi \in H^{\infty}$. Now there exist polynomials $\left\{p_{n}\right\}$ such that $M_{p_{n}}=p_{n}\left(M_{e}\right) \rightarrow M_{\psi}$ weakly and $\left\|M_{p_{n}}\right\|=\left\|p_{n}\right\|_{\infty} \leqq\|\psi\|_{\infty}$. (See [3, Solution 33].) In fact $p_{n}\left(M_{e} \oplus M_{e}\right) \rightarrow T_{1} \oplus T_{2}$ weakly and

$$
\left\|p_{n}\left(M_{e} \oplus M_{e}\right)\right\|=\left\|p_{n}\right\|_{\infty} \leqq\|\psi\|_{\infty} .
$$

Thus the boundedness condition is satisfied.

Proof of the Theorem. Suppose Lat $(V) \subseteq \operatorname{Lat}(B)$, where $V$ $=U_{s} \oplus U_{a} \oplus U_{+}$. Then $B=B_{1} \oplus B_{2} \oplus B_{3}$ with $\operatorname{Lat}\left(U_{s}\right) \subseteq \operatorname{Lat}\left(B_{1}\right)$ and Lat $\left(U_{a} \oplus U_{+}\right) \subseteq \operatorname{Lat}\left(B_{2} \oplus B_{3}\right)$. Hence $B_{1} \in Q_{U_{s}}$ and $B_{2} \oplus B_{3} \in Q_{U_{a} \oplus U_{+}}$ since $U_{b}$ is unitary, hence reflexive, and $U_{a} \oplus U_{+}$is reflexive by Lemma 3. But now $B=B_{1} \oplus B_{2} \oplus B_{3} \in Q_{U_{0} \oplus U_{a} \oplus U_{+}}=a_{V}$ by the corollary. Hence $V$ is reflexive.

We remark that the motivation for Lemma 2 was provided by Theorem 6 in [5] which states that if $U_{8}$ is a discrete (hence singular) unitary then there is a sequence of positive integers $N_{k} \rightarrow \infty$ such that $U_{s}^{N_{k}} \rightarrow I$ in the weak operator topology. From this it is trivial to conclude that $Q_{U_{s} \oplus U_{a}}=Q_{U_{b}} \oplus Q_{U_{a}}$ for any absolutely continuous $U_{a}$, since $U_{a}^{N_{k}} \rightarrow 0$ in the weak operator topology.

\section{REFERENCES}

1. R. G. Douglas, On the operator equation $S^{*} X T=X$ and related topics, Acta. Sci. Math. (Szeged) 30 (1969), 19-32.

2. P. R. Halmos, Introduction to Hilbert space and the theory of spectral multiplicity, Chelsea, New York, 1951. MR 13, 563. 
3. - A Hilbert space problem book, Van Nostrand, Princeton, N. J., 1967. MR 34 \#8178.

4. D. Sarason, Invariant subspaces and unstarred operator algebras, Pacific J. Math. 17 (1966), 511-517. MR 33 \#590.

5. J. Wermer, On invariant subspaces of normal operators, Proc. Amer. Math. Soc. 3 (1952), 270-277. MR 14, 55.

University of Michigan, Ann Arbor, Michigan 48104

UNIVERSITY OF KANSAS, LAWRENCE, KANSAS 66044 